PROCEEDINGS OF THE

AMERICAN MATHEMATICAL SOCIETY

Volume 132, Number 7, Pages 2141-2150

S 0002-9939(04)07360-5

Article electronically published on January 23, 2004

\title{
MYCIELSKI IDEAL AND THE PERFECT SET THEOREM
}

\author{
MIROSLAV REPICKÝ
}

(Communicated by Alan Dow)

\begin{abstract}
We make several observations on the Mycielski ideal and prove a version of the perfect set theorem concerning this ideal for analytic sets: If $A \subseteq \omega_{2}$ is an analytic set all projections of which are uncountable, then there is a perfect set $B \subseteq A$ a projection of which is the whole space. We also prove that (a modification of) an infinite game of Mycielski is determined for analytic sets.
\end{abstract}

\section{INTRODUCTION}

For $a \in[\omega]^{\omega}$ and $A \subseteq \omega_{2}$ let $\Gamma(A, a)$ be the infinite game for players I and II in which both players choose consecutive elements of a sequence $x \in{ }^{\omega} 2$. Player II chooses $x(n)$ for $n \in a$, and the other moves are done by player I. Player I wins if $x \in A$, and otherwise player II wins. This game was used by J. Mycielski [6] for introducing new invariant ideals on ${ }^{\omega_{2}} 2$ that are orthogonal to the ideals of sets of Lebesgue measure zero and of meager sets. A. Rosłanowski [8] introduced the next two ideals related to this game:

$$
\begin{gathered}
\mathfrak{C}_{2}=\left\{A \subseteq{ }^{\omega} 2:\left(\forall a \in[\omega]^{\omega}\right) \text { player II has a winning strategy in } \Gamma(A, a)\right\}, \\
\mathfrak{P}_{2}=\left\{A \subseteq{ }^{\omega} 2:\left(\forall a \in[\omega]^{\omega}\right) A\left\lceil a \neq{ }^{a} 2\right\},\right.
\end{gathered}
$$

where $A\left\lceil a=\left\{x\lceil a: x \in A\}\right.\right.$. The ideals $\mathfrak{C}_{2}, \mathfrak{P}_{2}$ are translation-invariant $\sigma$-ideals, $\mathfrak{P}_{2} \subseteq \mathfrak{C}_{2}$, every set in $\mathfrak{C}_{2}$ having the Baire property is meager, and every measurable set in $\mathfrak{C}_{2}$ has measure zero.

In this paper we make several observations on these two ideals and prove variants of the perfect set theorem for them (Theorems 2.2 and 3.2 ). In the case of the ideal $\mathfrak{C}_{2}$, this perfect set theorem is the determinacy of a modified game for analytic sets. Namely, let $\Gamma(A)$ be the following modification of the above game: At the beginning of the game, player I chooses $a \in[\omega]^{\omega}$, and then the players follow the rules of the game $\Gamma(A, a)$. Clearly,

$$
\mathfrak{C}_{2}=\left\{A \subseteq{ }^{\omega} 2 \text { : player II has a winning strategy in } \Gamma(A)\right\} .
$$

Theorem 3.2 says that the game $\Gamma(A)$ is determined for analytic sets $A \subseteq{ }^{\omega} 2$.

We use the standard notation. If $p \subseteq{ }^{<\omega} 2$ (or $\left.p \subseteq<\omega_{\omega}\right)$ is a tree and $s \in p$, then we denote $[p]=\left\{x:(\forall n) x\lceil n \in p\}\right.$ and $p_{s}=\{t \in p: s \subseteq t \vee t \subseteq s\}$. For $s \in{ }^{<\omega} 2$ or $s \in{ }^{<\omega} \omega,[s]$ denotes the corresponding clopen basic set $\{x: s \subset x\}$ in ${ }^{\omega} 2$ or ${ }^{\omega} \omega$.

Received by the editors August 21, 2002 and, in revised form, March 27, 2003.

2000 Mathematics Subject Classification. Primary 03E15; Secondary 03E17, 91A44.

Key words and phrases. Mycielski ideal, analytic sets, perfect set theorem.

This work was supported by a grant of Slovak Grant Agency VEGA 2/7555/20. 
Let us recall that $\mathfrak{s}$ is the minimal cardinality of a set $A \subseteq[\omega]^{\omega}$ such that for every $a \in[\omega]^{\omega}$ there is $b \in A$ such that $|a \backslash b|=|a \cap b|=\omega ; \mathfrak{h}$ is the least cardinal $\kappa$ such that $\mathcal{P}(\omega) /$ fin is not $\kappa$-distributive (see [5]).

\section{THE IDEAL $\mathfrak{P}_{2}$}

The ideal $\mathfrak{P}_{2}$ is called the Mycielski ideal (see [8] and [3]). We consider the following subideal $\mathcal{I}_{0}$ of $\mathfrak{P}_{2}$, defined by

$$
\mathcal{I}_{0}=\left\{A \subseteq{ }^{\omega} 2:\left(\forall a \in[\omega]^{\omega}\right)\left(\exists b \in[a]^{\omega}\right) \mid A\lceil b \mid \leq \omega\} .\right.
$$

This is the $\sigma$-ideal $\mathcal{I}_{\mathcal{X}}$ defined in [7] for the sequence of open sets $\mathcal{X}=\left\langle U_{n}: n \in \omega\right\rangle$ with $U_{n}=\left\{x \in \omega^{\omega}: x(n)=0\right\}$ where

$$
\mathcal{I}_{\mathcal{X}}=\left\{A \subseteq{ }^{\omega} 2:\left(\forall a \in[\omega]^{\omega}\right)\left(\exists b \in[a]^{\omega}\right) A \subseteq \bigcup_{m} \bigcap_{n \in b \backslash m} U_{n} \cup \bigcup_{m} \bigcap_{n \in b \backslash m}\left({ }^{\omega} 2 \backslash U_{n}\right)\right\} .
$$

\section{Theorem 2.1.}

(i) For every cardinal number $\kappa$ with $\omega<\kappa \leq \mathfrak{s}$,

$$
\mathcal{I}_{0}=\left\{A \subseteq{ }^{\omega} 2:\left(\forall a \in[\omega]^{\omega}\right)\left(\exists b \in[a]^{\omega}\right) \mid A\lceil b \mid<\kappa\} \subsetneq \mathfrak{P}_{2} .\right.
$$

(ii) $\mathfrak{s}=\min \left\{|A|: A \subseteq{ }^{\omega} 2\right.$ and $\left.A \in \mathfrak{P}_{2} \backslash \mathcal{I}_{0}\right\}$.

(iii) $\operatorname{add}\left(\mathcal{I}_{0}\right) \geq \mathfrak{h}$.

Proof. (i) The inclusions from left to right and the first equality are consequences of definitions. The only nontrivial part is the inequality $\subsetneq$. Consider a base matrix $B$ in $[\omega]^{\omega}$ (constructed in [1]). Every projection $B\left\lceil a\right.$ again is a base matrix in $[a]^{\omega}$ and has the size of the continuum. Therefore $B \in \mathfrak{P}_{2} \backslash \mathcal{I}_{0}$.

(ii) Every set of size $<\mathfrak{s}$ is in both $\mathfrak{P}_{2}$ and $\mathcal{I}_{0}$. If $\mathfrak{s}<\mathfrak{c}$, then any splitting family of size $\mathfrak{s}$ is in $\mathfrak{P}_{2} \backslash \mathcal{I}_{0}$. If $\mathfrak{s}=\mathfrak{c}$, then the set of the size of the continuum (base matrix) used in the proof of (i) belongs to $\mathfrak{P}_{2} \backslash \mathcal{I}_{0}$.

(iii) The inequality add $\left(\mathcal{I}_{0}\right) \geq \mathfrak{h}$ is due to the fact that $A \in \mathcal{I}_{0}$ if and only if the set $\left\{a \in[\omega]^{\omega}:(\forall x \in A)\left(\left(\forall^{\infty} n \in a\right) x(n)=0 \vee\left(\forall^{\infty} n \in a\right) x(n)=1\right)\right\}$ is open dense in $\mathcal{P}(\omega) /$ fin.

Another example of a set belonging to $\mathfrak{P}_{2} \backslash \mathcal{I}_{0}$ is constructed in Theorem 4.3

Now we prove a version of a perfect set theorem, which in a particular case says that if every projection of an analytic set in ${ }^{\omega_{2}} 2$ is uncountable, then it contains a perfect set a projection of which is the whole space.

Theorem 2.2. If an analytic subset of $\omega_{2}$ is not in $\mathcal{I}_{0}$, then it contains a perfect set that is not in $\mathfrak{P}_{2}$. Hence there is no analytic set in $\mathfrak{P}_{2} \backslash \mathcal{I}_{0}$.

Proof. Let $A \subseteq \omega_{2}$ be analytic, and let $A \notin \mathcal{I}_{0}$. Let $f$ be a continuous mapping from ${ }^{\omega} \omega$ onto $A$. Let $\left\{u_{n}: n \in \omega\right\}$ be an enumeration of $<\omega \omega$, and by induction on $n \in \omega$ let us construct a sequence of infinite sets $a_{n} \subseteq \omega$ such that

(i) $\left(\forall b \in\left[a_{0}\right]^{\omega}\right) \mid A\lceil b \mid>\omega$

(ii) $(\forall n \in \omega)\left(\mid f\left(\left[u_{n}\right]\right)\left\lceil a_{n}\left|\leq \omega \vee\left(\forall b \in\left[a_{n}\right]^{\omega}\right)\right| f\left(\left[u_{n}\right]\right)|b|>\omega\right)\right.$, and

(iii) $(\forall n \in \omega) a_{n+1} \subseteq a_{n}$.

Condition (i) is due to the assumption that $A \notin \mathcal{I}_{0}$, and condition (ii) is due to the fact that either $f\left(\left[u_{n}\right]\right)\left\lceil a_{n-1}\right.$ belongs to the ideal $\mathcal{I}_{0}$ (in ${ }^{a_{n-1}} 2$ ) or not. Let $a \in[\omega]^{\omega}$ be a pseudo-intersection of $\left\{a_{n}: n \in \omega\right\}$ and let $T=\{u \in<\omega \omega:|f([u])| a \mid>\omega\}$. For every $u \in T$ and every $b \in[a]^{\omega}$,

$$
f\left(\left[T_{u}\right]\right)\left\lceilb \supseteq f ( [ u ] ) \left\lceilb \backslash \bigcup \left\{ f([v])\left\lceil b: v \in \in^{<\omega} \omega \backslash T\right\},\right.\right.\right.
$$


and therefore

$$
(\forall u \in T)\left(\forall b \in[a]^{\omega}\right)\left|f\left(\left[T_{u}\right]\right)\right| b \mid>\omega .
$$

For $u \in T$ let $p^{(u)}=\left\{s \in \epsilon^{<\omega_{2}} 2:[s] \cap f\left(\left[T_{u}\right]\right) \neq \emptyset\right\}$, i.e., $\overline{f\left(\left[T_{u}\right]\right)}=\left[p^{(u)}\right]$. Then

$$
(\forall u \in T)\left(\forall^{\infty} n \in a\right)\left(\exists s_{1}, s_{2} \in p^{(u)} \cap^{n+1} 2\right) s_{1}(n) \neq s_{2}(n),
$$

since otherwise there exists an infinite set $b \subseteq a$ such that $\left|\left[p^{(u)}\right]\right| b \mid=1$, which contradicts (2.1). Condition (2.2) enables us to construct, by induction on $n=|s|$ for $s \in<\omega_{2}$, an infinite set $b=\left\{k_{n}: n \in \omega\right\} \subseteq a$ with $k_{n+1}>k_{n}$, and systems $X=\left\{u_{s}: s \in<^{<\omega} 2\right\} \subseteq T, Y=\left\{t_{s}: s \in{ }^{<\omega} 2\right\} \subseteq<\omega_{2}$ so that $u_{\emptyset}=\emptyset=t_{\emptyset}$, $t_{s \frown 0}, t_{s \frown 1} \in p^{\left(u_{s}\right)} \cap^{k_{n}+1} 2$ are such that $t_{s \frown i}\left(k_{n}\right)=i$ for $i=0,1$, and $u_{s \frown 0}, u_{s \frown 1} \in$ $T_{u_{s}}$ are such that $f\left(\left[T_{u_{s}{ }_{i}}\right]\right) \subseteq\left[t_{s} \frown i\right]$ for $s \in{ }^{n} 2$ and $i=0,1$. Clearly, $t_{s} \subseteq t_{s} \frown i$. The systems $X$ and $Y$ determine perfect trees in ${ }^{<\omega} \omega$ and ${ }^{<\omega} 2$, respectively, namely the trees

$$
T_{0}=\{u \in T:(\exists v \in X) u \subseteq v\}, \quad p_{0}=\left\{s \in{ }^{<\omega_{2}}:(\exists t \in Y) s \subseteq t\right\} .
$$

Hence the sets $B=\left[T_{0}\right]$ and $C=\left[p_{0}\right]$ are perfect compact, and clearly $C \subseteq A$, since $C=f(B)$. From the construction of $Y$ it follows that $C\left\lceil b={ }^{b} 2\right.$.

Corollary 2.3. The union of less than $\mathfrak{h}$ analytic sets from $\mathfrak{P}_{2}$ is in $\mathfrak{P}_{2}$.

Let $P$ be the set of all perfect trees in ${ }^{<\omega} 2$. For $n \in \omega$ and $p \in P$ we define $p \leq_{n} q$ if $p \subseteq q$ and $p \cap{ }^{n} 2=q \cap{ }^{n} 2$. A set $D \subseteq P$ is said to be $\omega$-dense if for every tree $p \in P$ and every $n \in \omega$ there is $q \in D$ such that $q \leq_{n} p$.

In [7] it was said that an ideal $\mathcal{I}$ on ${ }^{\omega} 2$ is tall if it contains all singletons and every perfect set $A \subseteq{ }^{\omega} 2$ has a perfect subset in $\mathcal{I}$. Let us recall that the condition in this definition concerning perfect sets is the so-called property $(\mathrm{P})$ introduced by M. Balcerzak and studied in a number of papers; see, e.g., [2] for references.

In 7 the question arose whether the ideals $\mathcal{I}_{\mathcal{X}}$ defined for various sequences of open sets are tall. The referee has kindly informed us that this is not true, because W. Dębski, J. Kleszcz, and Sz. Plewik in 4 have proved that the ideal $\mathfrak{P}_{2}$ (and hence also $\mathcal{I}_{0}$ ) does not have property $(\mathrm{P})$. Anyway, we can make several observations in this direction. Let us note that if $\mathcal{I}$ is a tall ideal on ${ }^{\omega} 2$, then for every $p \in P$ there exists $q \leq_{n} p$ such that $[q] \in \mathcal{I}$, i.e., the family of perfect trees $D_{\mathcal{I}}=\{p \in P:[p] \in \mathcal{I}\}$ is $\omega$-dense in $P$.

\section{Theorem 2.4.}

(i) The ideal $\mathcal{I}_{0}$ contains perfect sets. In fact, for every set $B \in \mathfrak{P}_{2}$ there is a perfect set $A \in \mathcal{I}_{0}$ disjoint from $B$.

(ii) For every perfect tree $p \subseteq{ }^{<\omega} 2$ there are a perfect tree $q \leq_{n} p$ and $c \in[\omega]^{\omega}$ such that for every $a \in[c]^{\omega}$ there is $b \in[a]^{\omega}$ such that $[q] \uparrow b$ is countable (hence $[q]\left\lceil c \in \mathcal{I}_{0}\right.$ inside ${ }^{c} 2$ ).

Proof. (i) Let $\left\{s_{n}: n \in \omega\right\}$ be the enumeration of $<\omega 2$ defined recursively by $s_{0}=\emptyset$, $s_{2 n+1}=s_{n}^{\frown} 0, s_{2 n+2}=s_{n}^{-1} 1$. The set

$$
\begin{aligned}
A=\left\{x \in{ }^{\omega} 2:(\forall n)[x(0)=1\right. & \wedge(x(n)=1 \rightarrow x(2 n+1)+x(2 n+2)=1) \\
& \wedge(x(n)=0 \rightarrow x(2 n+1)=x(2 n+2)=0)]\}
\end{aligned}
$$

is a perfect set (whose corresponding tree in ${ }^{<\omega_{2}} 2$ has exactly one branching node at each level), and the family $a_{x}=\{n: x(n)=1\}, x \in A$, is an almost disjoint family in $[\omega]^{\omega}$ coding all branches of $<\omega 2$ via the above enumeration. Hence for 
a given set $a \in[\omega]^{\omega}$ either $b=a$ is almost disjoint from every $a_{x}, x \in A$, and then $(\forall x \in A)\left(\forall^{\infty} n \in b\right) x(n)=0$, or there is $x_{0} \in A$ such that $b=a \cap a_{x_{0}}$ is infinite, and then $\left(\forall x \in A, x \neq x_{0}\right)\left(\forall^{\infty} n \in b\right) x(n)=0$; hence $A\lceil b$ is countable.

Now we prove that for every set $B \in \mathfrak{P}_{2}$ there is a perfect set $A \in \mathcal{I}_{0}$ disjoint from $B$. Let $a \in[\omega]^{\omega}$ be a set with infinite complement such that $B\left\lceil a \neq{ }^{a} 2\right.$, and let $y \in{ }^{\omega_{2}}$ be such that $y\left\lceil a \notin B\left\lceil a\right.\right.$. Let $\pi:{ }^{\omega_{2}} \rightarrow{ }^{\omega}{ }^{\omega} a_{2}$ be the homeomorphism induced by the one-to-one mapping from $\omega$ onto $\omega \backslash a$. Let $A_{0} \in \mathcal{I}_{0}$ be a perfect subset of ${ }^{\omega} 2$. Then the set $A=\pi\left(A_{0}\right) \times\{y\lceil a\}$ is perfect, $A$ is disjoint from $B$, and $A \in \mathcal{I}_{0}$.

(ii) If $[p] \in \mathfrak{P}_{2}$, then $[p] \in \mathcal{I}_{0}$ and the assertion holds for $q=p$ and $c=\omega$. The case $[p] \notin \mathfrak{P}_{2}$ is nontrivial. First let us prove the following weaker property:

(2.3) For every perfect set $B \notin \mathfrak{P}_{2}$ in ${ }^{\omega} 2$ there are a perfect set $A \subseteq B$ and $c \in[\omega]^{\omega}$ such that for every $a \in[c]^{\omega}$ there is $b \in[a]^{\omega}$ such that $A\lceil b$ is countable.

Since $B \notin \mathfrak{P}_{2}$, there is a set $c \in[\omega]^{\omega}$ such that $B\left\lceil c={ }^{c} 2\right.$. By assertion (i) there exists a perfect set $C \subseteq{ }^{c} 2$ such that for every $a \in[c]^{\omega}$ there is $b \in[a]^{\omega}$ such that $C\lceil b$ is countable. The set $\{x \in B: x\lceil c \in C\}$ is an uncountable closed set; let $A$ be a perfect subset of it.

Let us assume that $[p] \notin \mathfrak{P}_{2}$. Let $\left\{s_{i}: i \leq m\right\}=p \cap^{n} 2$. By induction, using (2.3), we can find infinite subsets of $c_{-1}=\omega, c_{0} \supseteq c_{1} \supseteq \cdots \supseteq c_{m}$ and perfect trees $q_{i} \leq p_{s_{i}}$ so that if $\left[p_{s_{i}}\right]\left\lceil c_{i-1} \in \mathfrak{P}_{2}\right.$ in ${ }^{c_{i-1}} 2$, then $c_{i}=c_{i-1}$ and $q_{i}=p_{s_{i}}$, and if $\left[p_{s_{i}}\right]\left\lceil c_{i-1} \notin \mathfrak{P}_{2}\right.$ in ${ }^{c_{i-1}} 2$, then $q_{i} \leq p_{s_{i}}$ and $c_{i}$ are such that $\left(\forall a \in\left[c_{i}\right]^{\omega}\right)\left(\exists b \in[a]^{\omega}\right)\left|\left[q_{i}\right]\right| b \mid \leq \omega$. Let $c=c_{m}$ and $q=\bigcup_{i \leq m} q_{i}$.

Next we present some perfect sets $A \subseteq{ }^{\omega_{2}} 2$, discerning the case when almost all projections are countable from the case when almost every projection is the whole space.

\section{Fact 2.5.}

(i) For a finite partition $\mathcal{A}$ of $\omega$ there is a perfect set $A \subsetneq{ }^{\omega} 2$ such that $A\left\lceil a={ }^{a} 2\right.$ for all $a \in \mathcal{A}$.

(ii) There is a perfect set $A \subseteq \omega_{2}$ such that $\left(\forall a \in[\omega]^{\omega}\right)\left(\exists b \in[a]^{\omega}\right) A\left\lceil b={ }^{b} 2\right.$ and there is no finite partition $\mathcal{A}$ of $\omega$ into infinite sets such that $A\left\lceil a={ }^{a} 2\right.$ for all $a \in \mathcal{A}$.

(iii) There is a perfect set $A \subseteq{ }^{\omega} 2$ such that for some set $c \in[\omega]^{\omega}$ the following conditions hold true:

(a) $|A| c \mid>\omega$ and $\left(\forall a \in[c]^{\omega}\right)\left(\exists b \in[a]^{\omega}\right) \mid A\lceil b \mid \leq \omega$,

(b) $\left(\forall a \in[\omega \backslash c]^{\omega}\right)\left(\exists b \in[a]^{\omega}\right) A\left\lceil b={ }^{b} 2\right.$, and

(c) $A\left\lceil(\omega \backslash c) \neq \omega \backslash c_{2}\right.$ and there is no finite partition $\mathcal{A}$ of $\omega \backslash c$ into infinite sets such that $A\left\lceil a={ }^{a} 2\right.$ for all $a \in \mathcal{A}$.

(iv) There is a perfect set $A \subseteq \omega_{2}$ such that there is no set $c \in \mathcal{P}(\omega)$ such that

(a) $\left(\forall a \in[c]^{\omega}\right)\left(\exists b \in[a]^{\omega}\right) \mid A\lceil b \mid \leq \omega$, and

(b) $\left(\forall a \in[\omega \backslash c]^{\omega}\right)\left(\exists b \in[a]^{\omega}\right) A\left\lceil b={ }^{b} 2\right.$.

Proof. (i) For $a \in \mathcal{A}$ let $e_{a}: \omega \rightarrow a$ be a one-to-one mapping from $\omega$ onto $a$. Let $A=\left\{x \in \omega^{\omega}:(\forall a, b \in \mathcal{A})(\forall n \in \omega) x\left(e_{a}(n)\right)=x\left(e_{b}(n)\right)\right\}$.

(ii) Instead of $\omega$ we consider $\omega \times \omega$. Let us denote

$$
\begin{aligned}
A & =\left\{x \in{ }^{\omega \times \omega_{2}}:(\forall i, j, n) x(i, n)=x(j, n) \vee x(i, n)=x(j, n+1)\right\} \\
& =\left\{x \in{ }^{\omega \times \omega_{2}} 2:(\forall j, n) x(j, n)=x(j, n+1) \rightarrow(\forall i) x(i, n)=x(j, n)\right\} .
\end{aligned}
$$


$A$ is a perfect subset of $\omega \times \omega 2$.

For every $i \in \omega, A \uparrow(\{i\} \times \omega)=\{i\} \times \omega$. To see this, let $x_{0} \in\{i\} \times \omega_{2}$ be arbitrary. Define $x \in A$ with $x_{0} \subseteq x$ by $x(j, n)=x_{0}(i, n)$ for all $j, n \in \omega$.

Furthermore, we show that if $a \subseteq \omega \times \omega$ is a function, then $A\left\lceil a={ }^{a} 2\right.$. Let $a \subseteq \omega \times \omega$ be a function. Without loss of generality, let us assume that $\operatorname{dom}(a)=\omega$. Let $x_{0} \in{ }^{a} 2$. There is $x \in{ }^{\omega \times \omega_{2}} 2, x \supseteq x_{0}$, defined by $x(i, n)=x_{0}(i, a(i))$ if $n-a(i)$ is even and $x(i, n)=1-x_{0}(i, a(i))$ if $n-a(i)$ is odd. Then $x \in A$, since $x(j, n) \neq x(j, n+1)$ for all $j, n \in \omega$.

Now we can see that $\left(\forall a \in[\omega \times \omega]^{\omega}\right)\left(\exists b \in[a]^{\omega}\right) A\left\lceil b={ }^{b} 2\right.$. This is because either for some $n$ the set $b=a \cap(\{n\} \times \omega)$ is infinite, or in the opposite case the set $b=\{(i, n) \in a:(\forall m<n)(i, m) \notin a\}$ is an infinite function and by previous cases $A \nmid b={ }^{b} 2$.

We now prove that there is no finite partition $\mathcal{A}$ of $\omega \times \omega$ such that $A\left\lceil a={ }^{a} 2\right.$ for every $a \in \mathcal{A}$. So let us assume that $\mathcal{A}$ is a partition of $\omega \times \omega$ and $|\mathcal{A}|=k$. By the Ramsey theorem $\omega \rightarrow(\omega)_{k}^{2}$, there is an infinite set $b \subseteq \omega$ such that $\{(m, n) \in b \times b$ : $m>n\} \subseteq a_{0}$ for some $a_{0} \in \mathcal{A}$. We show that for $a=\{(m, n) \in b \times b: m>n\}$ we have $A\left\lceil a \neq{ }^{a} 2\right.$, and hence $A\left\lceil a_{0} \neq{ }^{a_{0}} 2\right.$. Let $i>j>m>n$ be fixed elements of $b$ and let $x_{0} \in{ }^{a} 2$ be any function such that $x_{0}(i, n) \neq x_{0}(j, n)=0$, and

$$
x_{0}(i, m)= \begin{cases}x_{0}(i, n) & \text { if } m-n \text { is odd } \\ x_{0}(j, n) & \text { if } m-n \text { is even }\end{cases}
$$

Let us assume that there is an $x \in A$ such that $x \supseteq x_{0}$. Since $x(i, n) \neq x(j, n)$, then $x(i, n) \neq x(i, n+1), x(j, n) \neq x(j, n+1)$, and consequently $x(i, n+1) \neq x(j, n+1)$. Using the same argument again, we see that $x(i, n)=x(i, n+2)$, and by induction on $p \in \omega$ we get $x(i, n)=x(i, n+2 p) \neq x(i, n+2 p+1)$, which contradicts (2.4). Therefore $A\left\lceil a_{0} \neq a_{0} 2\right.$.

(iii) Instead of $\omega$ we consider the countable ordinal $\omega+\omega$ and the countable interval of ordinals $[\omega, \omega+\omega)$. Let $A_{1}$ be a perfect set such that $A_{1} \in \mathcal{I}_{0}$, and let $A_{2} \subseteq[\omega, \omega+\omega) 2$ be the perfect set from case (ii). Then $A=\left\{x \in{ }^{\omega+\omega} 2: x\left\lceil\omega \in A_{1}\right.\right.$ and $\left.x \uparrow[\omega, \omega+\omega) \in A_{2}\right\}$, as a perfect subset of $\omega+\omega 2$, has the required property, with $\omega+\omega$ instead of $\omega$ and with $c=\omega$.

(iv) Let us consider the set $\omega \times \omega$ instead of the set $\omega$, and let

$$
A=\left\{x \in{ }^{\omega \times \omega_{2}}:(\forall n, m \in \omega) x(n, m+1)=x(n, m)\right\} .
$$

Let $a_{n}=\{(n, m): m \in \omega\}$. Clearly, $|A| a_{n} \mid=1$. Let us assume that there is a set $c \in \mathcal{P}(\omega \times \omega)$ satisfying (a) and (b). Then, according to (b), for every $n$, $c$ contains almost all elements of $a_{n}$. Let $a=\{(n, m) \in c:(\forall k<m)(n, k) \notin c\}$. Then $A\left\lceil a={ }^{a} 2\right.$. But since $a \subseteq c$, this proves that $c$ does not satisfy condition (a), a contradiction.

\section{The IDEAL $\mathfrak{C}_{2}$}

For $a \in[\omega]^{\omega}$ let

$$
\mathfrak{C}_{2, a}=\left\{A \subseteq{ }^{\omega} 2:\left(\forall b \in[a]^{\omega}\right) \text { player II has a winning strategy in } \Gamma(A, b)\right\} .
$$

Clearly, $\mathfrak{C}_{2, a}$ is a $\sigma$-ideal and $\mathfrak{C}_{2}=\mathfrak{C}_{2, a} \cap \mathfrak{C}_{2, \omega \backslash a}$ for every $a \in[\omega]^{\omega}$. The following holds true: 
Lemma 3.1. If player II has no winning strategy in $\Gamma(A, a)$ and $B \in \mathfrak{C}_{2, a}$, then for every $b \in[a]^{\omega}$ with infinite $b^{\prime}=a \backslash b$, player II has no winning strategy in $\Gamma(A \backslash B, b)$.

Proof. Let us assume the lemma fails for $b \subseteq a$. Then there are winning strategies $S$ and $S^{\prime}$ for player II in $\Gamma(A \backslash B, b)$ and $\Gamma\left(B, b^{\prime}\right)$, respectively. Then the strategy of player II which follows $S$ for $n \in b$ and $S^{\prime}$ for $n \in b^{\prime}$ is a winning strategy of player II in $\Gamma(A, a)$, a contradiction.

Notice that player I has a winning strategy in the game $\Gamma(A)$ if and only if there are an infinite set $a \subseteq \omega$ and a perfect tree $p \subseteq{ }^{<\omega} 2$ with $[p] \subseteq A$ in which every branch ramifies on all levels $n \in a$. It turns out that the analogue of the perfect set theorem corresponding to Theorem 2.2 for this ideal is the determinacy of the game $\Gamma(A)$ for analytic sets:

Theorem 3.2. If $A$ is an analytic subset of $\omega_{2}$ such that $A \notin \mathfrak{C}_{2, a}$, then there exists $b \in[a]^{\omega}$ such that player I has a winning strategy in the game $\Gamma(A, b)$. In particular, the game $\Gamma(A)$ is determined for analytic sets $A \subseteq{ }^{\omega} 2$.

Proof. Let $A \subseteq{ }^{\omega} 2$, and let $f$ be a continuous mapping from ${ }^{\omega} \omega$ onto $A$. Let us assume that $A \notin \mathfrak{C}_{2, a}$, and let $a_{0} \subseteq a$ be such that II has no winning strategy in $\Gamma\left(A, a_{0}\right)$. Let $\left\{u_{n}: n \in \omega\right\}$ be an enumeration of $<\omega \omega$ with $u_{0}=\emptyset$, and by induction on $n \in \omega$ let us construct infinite sets $a_{n+1} \subseteq a_{n}$ such that for every $n \in \omega$, either $f\left(\left[u_{n}\right]\right) \in \mathfrak{C}_{2, a_{n}}$, or player II has no winning strategy in $\Gamma\left(f\left(\left[u_{n}\right]\right), a_{n}\right)$.

Let $a^{\prime} \subseteq a$ be an infinite pseudo-intersection of the system $\left\{a_{n}: n \in \omega\right\}$. It follows that player II has no winning strategy in $\Gamma\left(A, a^{\prime}\right)$, and for every $n \in \omega$, either $f\left(\left[u_{n}\right]\right) \in \mathfrak{C}_{2, a^{\prime}}$, or there is $m \in \omega$ such that player II has no winning strategy in $\Gamma\left(f\left(\left[u_{n}\right]\right), a^{\prime} \backslash m\right)$.

Let $T=\left\{u \in{ }^{<\omega} \omega: f([u]) \notin \mathfrak{C}_{2, a^{\prime}}\right\}$. We have

$$
f\left(\left[T_{u}\right]\right) \supseteq f([u]) \backslash \bigcup\left\{f([v]): v \in<\omega 2 \text { and } f([v]) \in \mathfrak{C}_{2, a^{\prime}}\right\} .
$$

Let $a^{\prime \prime} \in\left[a^{\prime}\right]^{\omega}$ be such that $a^{\prime} \backslash a^{\prime \prime}$ is infinite. Since $\mathfrak{C}_{2, a^{\prime}}$ is a $\sigma$-ideal, using Lemma3.1 we can easily see that

(3.1) $\quad(\forall u \in T)(\exists m \in \omega)$ player II has no winning strategy in $\Gamma\left(f\left(\left[T_{u}\right]\right), a^{\prime \prime} \backslash m\right)$.

For $u \in T$ let $p^{(u)}=\left\{s \in{ }^{<\omega} 2:[s] \cap f\left(\left[T_{u}\right]\right) \neq \emptyset\right\}$. By induction on $n=|s|$ for $s \in{ }^{<\omega} 2$ we define an infinite set $b=\left\{k_{n}: n \in \omega\right\} \subseteq a^{\prime \prime}$ with $k_{n+1}>k_{n}$ and systems $X=\left\{u_{s}: s \in{ }^{<\omega} 2\right\} \subseteq T, Y=\left\{t_{s}: s \in{ }^{<\omega} 2\right\} \subseteq{ }^{<\omega_{2}} 2$ so that $u_{\emptyset}=\emptyset$, and for every $s \in<\omega_{2}$,

(i) if $|s|=n$, then $t_{s} \in p^{\left(u_{s}\right)} \cap{ }^{k_{n}} 2$,

(ii) $t_{s} 0, t_{s} 1 \in p^{\left(u_{s}\right)}$, and

(iii) $f\left(\left[T_{u_{s} \frown i}\right]\right) \subseteq\left[t_{s} i\right]$ for $i=0,1$.

Let us assume that $u_{s}$ for $s \in{ }^{n} 2$ have been constructed. By (3.1) there is $m$ such that player II has no winning strategy in any game $\Gamma\left(f\left(\left[T_{u_{s}}\right]\right), a^{\prime \prime} \backslash m\right)$ for all $s \in{ }^{n} 2$. If $n>0$, we take such $m>k_{n-1}$. Let $k_{n}=\min \left(a^{\prime \prime} \backslash m\right)$. Then for every $s \in{ }^{n} 2$ there is $t_{s} \in p^{\left(u_{s}\right)} \cap{ }^{k_{n}} 2$ such that $t_{s}^{\frown} 0, t_{s}^{\frown 1 \in p^{\left(u_{s}\right)}}$ (otherwise II wins in his first move). Hence conditions (i) and (ii) are fulfilled. Using the continuity of $f$, there are $u_{s \frown 0}$ and $u_{s>1}$ so that (iii) is fulfilled.

Clearly, $t_{s} \subseteq t_{s} i \subseteq t_{s} \frown i$ and $u_{s} \subseteq u_{s} \frown i$, and the systems $X$ and $Y$ determine perfect trees $T_{0}$ and $p_{0}$ in ${ }^{<\omega} \omega$ and ${ }^{<\omega_{2}}$, respectively. The corresponding perfect sets $B=\left[T_{0}\right], C=\left[p_{0}\right]$ are compact, and, since $C=f(B), C \subseteq A$. The perfect 
tree $p_{0}$ has the property that a node in $p_{0}$ is a branching node if and only if it is on the level $k_{n}$ for some $n$. Hence the winning strategy of player I in $\Gamma(A, b)$ is to follow any branch in the tree $p_{0}$.

The following lemma has no particular importance, but we need it in the proof of Theorem 4.4 (namely Claim 4.7).

Lemma 3.3. If $p \subseteq{ }^{<\omega} 2$ is a perfect tree in which the value 1 appears only at branching nodes and $a \in[\omega]^{\omega}$ is such that for every $t \in p,\left[p_{t}\right]\lceil a$ has a nonempty interior in ${ }^{a} 2$, then there is $b \in[a]^{\omega}$ such that player I has a winning strategy in $\Gamma([p], b)$.

Proof. For every $t \in p$, since $\left[p_{t}\right]\left\lceil a\right.$ has a nonempty interior in ${ }^{a} 2$, for all but finitely many $n \in a$ there is $t^{\prime} \in p \cap^{n-1} 2$ with $t^{\prime} \supseteq t$ such that $t^{\prime}-1 \in p$. Since the value 1 appears in $p$ only at branching nodes, for all but finitely many $n \in a$ there is a branching node $t^{\prime} \in p \cap^{n-1} 2, t^{\prime} \supseteq t$. Therefore, by induction we can construct an increasing sequence $k_{n}, n \in \omega$, and $t_{s} \in p \cap^{k_{n}-1} 2$ for $s \in{ }^{n} 2$ such that $t_{s} i \subseteq t_{s} \frown i$ for $i=0,1$. Let $b=\left\{k_{n}: n \in \omega\right\}$. Then the strategy of player I to follow any branch in the tree generated by the system $\left\{t_{s}: s \in{ }^{<\omega} 2\right\}$ is a winning strategy in the game $\Gamma([p], b)$.

\section{ISOMETRIC IMAGES OF SETS}

In what follows, by a metric on the Cantor space ${ }^{\omega} 2$ we mean the metric $d$ defined by $d(x, y)=2^{-\min \{n: x(n) \neq y(n)\}}$ for $x, y \in{ }^{\omega} 2, x \neq y$. Let $\mathcal{F}$ be the family of all bijective mappings $\varphi:{ }^{<\omega} 2 \rightarrow{ }^{<\omega} 2$ such that for every $s, t \in{ }^{<\omega} 2$,

(i) $|\varphi(s)|=|s|$, and

(ii) $s \subseteq t$ implies $\varphi(s) \subseteq \varphi(t)$.

Every isometric function $f:{ }^{\omega} 2 \rightarrow{ }^{\omega} 2$ is a homeomorphism of the form $f_{\varphi}$ where $f_{\varphi}(x)=\bigcup_{n \in \omega} \varphi\left(x\lceil n)\right.$. Clearly, $f_{\varphi}^{-1}=f_{\varphi^{-1}}$.

Lemma 4.1. If $A, B \subseteq{ }^{\omega} 2$ and $B$ is isometric to $A$, then for every $a \in[\omega]^{\omega}$, player I (player II) has a winning strategy in $\Gamma(A, a)$ if and only if he has one in $\Gamma(B, a)$. Consequently, $A \in \mathfrak{C}_{2}$ if and only if $B \in \mathfrak{C}_{2}$.

Proof. Let $B=f_{\varphi}(A)$ for some $\varphi \in \mathcal{F}$, and let $F:<\omega_{2} \rightarrow\{0,1\}$ be a winning strategy for player I or player II in the game $\Gamma(A, a)$ where $a \in[\omega]^{\omega}$. Then the function $F^{\prime}(s)=\varphi\left(\varphi^{-1}(s)^{\frown} F\left(\varphi^{-1}(s)\right)\right)(n), s \in{ }^{n} 2, n \in \omega$, is a winning strategy for the same player in $\Gamma(B, a)$, because if $x(n) \neq F\left(x\lceil n)\right.$, then $f_{\varphi}(x)(n)=$ $\varphi\left(x\lceil n \frown x(n))(n) \neq \varphi\left(x\left\lceil n \frown F(x\lceil n))(n)=F^{\prime}\left(\varphi(x\lceil n))=F^{\prime}\left(f_{\varphi}(x)\lceil n)\right.\right.\right.\right.\right.$.

Theorem 4.2. The ideal $\mathfrak{P}_{2}$ is not closed under isometric images. In fact, every uncountable analytic set in ${ }^{\omega} 2$ is isometric to a set that does not belong to $\mathfrak{P}_{2}$.

Proof. Since every uncountable analytic set contains a perfect subset, it is enough to prove the theorem for perfect sets. Clearly, every perfect set $A \subseteq{ }^{\omega} 2$ is isometric to a perfect set $B \subseteq{ }^{\omega} 2$ such that in its corresponding perfect tree $p \subseteq{ }^{<\omega} 2$ the branches have the same values at all nodes between any two successive points of ramification. Then, as in the proof of Theorem 3.3 of $\left[8\right.$, let $a=\left\{n_{0}, n_{1}, \ldots\right\}$ be an increasing sequence of natural numbers such that for every $s \in{ }^{n_{i}+1} 2 \cap p$ there is a branching node above $s$ before level $n_{i+1}$, and then $B\left\lceil a \supseteq[p]\left\lceil a={ }^{a} 2\right.\right.$. 
If a set $A \subseteq{ }^{\omega} 2$ has size $<\mathfrak{c}$, then all its isometric images belong to $\mathfrak{P}_{2}$. This fact together with the previous theorem suggests the question of whether there is a set $A \subseteq{ }^{\omega} 2$ of size $\mathfrak{c}$ all isometric images of which belong to $\mathfrak{P}_{2}$. The next theorem partially answers this question.

Theorem 4.3. If the covering number of the ideal of meager sets or of the ideal of measure zero sets is $\mathfrak{c}$, then there is a subset of $2^{\omega}$ of size $\mathfrak{c}$ every isometric image of which belongs to $\mathfrak{P}_{2} \backslash \mathcal{I}_{0}$.

Proof. Let $\left\{\left(a_{\alpha}, \varphi_{\alpha}\right): \alpha<\mathfrak{c}\right\}$ be an enumeration of $[\omega]^{\omega} \times \mathcal{F}$. Since the sets $A_{x, a, \varphi}=\left\{y \in{ }^{\omega} 2: f_{\varphi}(y)\left\lceil a=f_{\varphi}(x)\lceil a\}\right.\right.$, for $x \in{ }^{\omega} 2, a \in[\omega]^{\omega}$ and $\varphi \in \mathcal{F}$, are meager sets of measure zero, using the assumption of the theorem on the covering number of such sets, we can inductively define reals $x_{\alpha}, y_{\alpha}$ for $\alpha<\mathfrak{c}$ so that

$$
x_{\alpha} \in{ }^{\omega} 2 \backslash \bigcup\left\{A_{x_{\beta}, a_{\gamma}, \varphi_{\gamma}} \cup A_{y_{\beta}, a_{\gamma}, \varphi_{\gamma}}: \beta, \gamma<\alpha\right\},
$$

and

$$
y_{\alpha} \in{ }^{\omega} 2 \backslash \bigcup\left\{A_{x_{\beta}, a_{\gamma}, \varphi_{\gamma}} \cup A_{y_{\beta}, a_{\gamma}, \varphi_{\gamma}} \cup A_{x_{\alpha}, a_{\gamma}, \varphi_{\gamma}}: \beta, \gamma<\alpha\right\} .
$$

Let $A=\left\{x_{\alpha}: \alpha<\mathfrak{c}\right\}$. If $\alpha>\beta>\gamma$, then $x_{\alpha} \notin A_{x_{\beta}, a_{\gamma}, \varphi_{\gamma}}$ and hence $f_{\varphi_{\gamma}}\left(x_{\alpha}\right)\left\lceil a_{\gamma} \neq\right.$ $f_{\varphi_{\gamma}}\left(x_{\beta}\right)\left\lceil a_{\gamma}\right.$. Therefore, $\mid f_{\varphi_{\gamma}}(A)\left\lceil a_{\gamma} \mid=\mathfrak{c}\right.$ for every $\gamma$. Also, $f_{\varphi_{\gamma}}\left(y_{\gamma}\right)\left\lceil a_{\gamma} \notin f_{\varphi_{\gamma}}(A)\left\lceil a_{\gamma}\right.\right.$ for every $\gamma$, since $f_{\varphi_{\gamma}}\left(y_{\gamma}\right)\left\lceil a_{\gamma} \neq f_{\varphi_{\gamma}}\left(x_{\alpha}\right)\left\lceil a_{\gamma}\right.\right.$ by (4.1), for $\alpha>\gamma$, and by (4.2) for $\alpha \leq \gamma$.

Theorem 4.4. Let $A \subseteq \omega_{2} 2$ be a closed set. Then $A \in \mathfrak{C}_{2}$ if and only if

$$
\left(\forall a \in[\omega]^{\omega}\right)\left(\exists b \in[a]^{\omega}\right)(\exists \varphi \in \mathcal{F})\left|f_{\varphi}(A)\right| b \mid \leq \omega .
$$

Proof. If $b \in[\omega]^{\omega}$ and $\varphi \in \mathcal{F}$ are such that $f_{\varphi}(A)\lceil b$ is countable, then there is a winning strategy for II in $\Gamma\left(f_{\varphi}(A), b\right)$. By Lemma 4.1, then there is a winning strategy for II in $\Gamma(A, b)$. Therefore the condition in the theorem implies that $A \in \mathfrak{C}_{2}$.

Conversely, let $A \in \mathfrak{C}_{2}$ and $a \in[\omega]^{\omega}$ be arbitrary. Let $p \subseteq<\omega 2$ be the tree such that $A=[p]$. We find $b \in[a]^{\omega}$ and $\varphi \in \mathcal{F}$ such that $f_{\varphi}(A)\lceil b$ is countable.

By induction on $n \in \omega$ let us define trees $p_{n} \subseteq p$ and $a_{n} \in[a]^{\omega}$ with $p_{n+1} \subseteq p_{n}$ and $a_{n+1} \subseteq a_{n}$ as follows: Let $p_{0}=p$ and $a_{0}=a$. Let us assume that $p_{n}$ and $a_{n}$ are defined. If there are $s \in p_{n}$ and $c \in\left[a_{n}\right]^{\omega}$ such that for some $\varphi \in \mathcal{F},\left[\varphi\left(\left(p_{n}\right)_{s}\right)\right]\lceil c$ is countable let $s_{n}$ be such an $s$ of minimal length, let $a_{n+1}$ be the corresponding set $c$, and let $p_{n+1}=p_{n} \backslash\left\{s \in p_{n}: s \supseteq s_{n}\right\}$. If there is no such $s \in p_{n}$ (e.g., if $p_{n}=\emptyset$ ), then let $p_{n+1}=p_{n}$ and $a_{n+1}=a_{n}$.

Let $b \subseteq a$ be an infinite pseudo-intersection of all $a_{n}, n \in \omega$, and let $\omega_{p} \leq \omega$ be the maximal ordinal such that $p_{n} \neq p_{n+1}$ for all $n<\omega_{p}$. Thus, for every $n<\omega_{p}$, $s_{n}$ is defined and

$$
\text { there is } \varphi_{n} \in \mathcal{F} \text { such that }\left[\varphi_{n}\left(\left(p_{n}\right)_{s_{n}}\right)\right]\lceil b \text { is countable. }
$$

For any $m \in \omega$ and $\varphi \in \mathcal{F}$ there is $\varphi_{n} \in \mathcal{F}$ such that (4.4) holds and $\varphi_{n}(s)=\varphi(s)$ for $|s| \leq m$.

Claim 4.5. $\left|s_{n}\right| \leq\left|s_{n+1}\right|$ whenever $n+1<\omega_{p}$.

Proof. Let $m=\max \left\{\left|s_{n}\right|,\left|s_{n+1}\right|\right\}$ and let $\varphi_{n}, \varphi_{n+1} \in \mathcal{F}$ be such that $\varphi_{n}(s)=$ $\varphi_{n+1}(s)=s$ for $|s| \leq m$ and (4.4) holds for $n$ and $n+1$. Then $\left(\varphi_{n} \uparrow\left(p_{n}\right)_{s_{n}}\right) \cup$ $\left(\varphi_{n+1} \uparrow\left(p_{n+1}\right)_{s_{n+1}}\right)$ is a function, and hence it can be extended to a $\varphi \in \mathcal{F}$. Clearly, $\left[\varphi\left(\left(p_{n}\right)_{s_{n}} \cup\left(p_{n+1}\right)_{s_{n+1}}\right)\right]\left\lceil c=\left[\varphi_{n}\left(\left(p_{n}\right)_{s_{n}}\right)\right]\left\lceil c \cup\left[\varphi_{n+1}\left(\left(p_{n+1}\right)_{s_{n+1}}\right)\right]\lceil c\right.\right.$ is countable. 
Since $p_{n}=\left(p_{n}\right)_{s_{n}} \cup p_{n+1}$, we have $\left(p_{n}\right)_{s_{n+1}} \subseteq\left(p_{n}\right)_{s_{n}} \cup\left(p_{n+1}\right)_{s_{n+1}}$, and hence the inequality $\left|s_{n+1}\right|<\left|s_{n}\right|$ would contradict the minimality of $\left|s_{n}\right|$.

Let $q=\bigcap_{n \in \omega} p_{n}$.

Claim 4.6. If $q \neq \emptyset$, then $q$ is a tree, and for any $s \in q, c \in[b]^{\omega}$, and $\varphi \in \mathcal{F}$, the projection $\left[\varphi\left(q_{s}\right)\right]\lceil c$ is uncountable.

Proof. If $\omega_{p}<\omega$, then there is $n$ such that $q=p_{n}=p_{n+1}$, and the claim holds by the definition of $p_{n+1}$.

,Let $\omega_{p}=\omega$. To obtain a contradiction, let us assume that for some $t \in q$, $c \in[b]^{\omega}$, and $\varphi \in \mathcal{F}$, the projection $\left[\varphi\left(q_{s}\right)\right]\lceil c$ is countable. Let us fix an $m$ such that $|t|<\left|s_{m}\right|$, and let $\varphi_{n} \in \mathcal{F}$ for $n \in \omega$ be such that $\varphi_{n}(s)=\varphi(s)$ for $|s| \leq\left|s_{n}\right|$ and (4.4) holds for all $n$. By Claim 4.5 $s_{n}, n \in \omega$, are pairwise incompatible, and hence $\left(\varphi\lceil q) \cup \bigcup_{n \in \omega}\left(\varphi_{n}\left\lceil\left(p_{n}\right)_{s_{n}}\right)\right.\right.$ is a function. Let $\psi \in \mathcal{F}$ extend it. Now $p=q \cup \bigcup_{n \in \omega}\left(p_{n}\right)_{s_{n}}, t \in q \subseteq p_{m}$, and $\left(p_{m}\right)_{t} \subseteq q_{t} \cup \bigcup_{n \in \omega}\left(p_{n}\right)_{s_{n}}$. Therefore, $\left[\psi\left(\left(p_{m}\right)_{t}\right)\right]\left\lceil c \subseteq\left[\varphi\left(q_{t}\right)\right]\left\lceil c \cup \bigcup_{n \in \omega}\left[\varphi_{n}\left(\left(p_{n}\right)_{s_{n}}\right)\right]\lceil c\right.\right.$ is countable, which contradicts the minimality of $\left|s_{m}\right|$.

Claim 4.7. $q=\emptyset$.

Proof. Let us assume that $q \neq \emptyset$, and let us define $\varphi \in \mathcal{F}$ by induction as follows:

(1) $\varphi(\emptyset)=\emptyset$.

(2) If for some $i \in\{0,1\}$ we have $s \frown i \in q$ and $s \frown(1-i) \notin q$, then $\varphi(s \frown i)=$ $\varphi(s) \frown 0$ and $\varphi(s \frown(1-i))=\varphi(s) \frown 1$.

(3) Otherwise, $\varphi_{q}(s \frown i)=\varphi(s) \frown i$ for $i=0,1$.

It follows that in the tree $q^{\prime}=\varphi(q)$ the value 1 appears only at branching nodes. Using Claim [4.6, we can construct a centered system $\left\{c_{s}: s \in q^{\prime}\right\} \subseteq[b]^{\omega}$ such that $\left[q_{s}^{\prime}\right]\left\lceil c_{s}={ }^{c_{s}} 2\right.$, and let $c \subseteq b$ be an infinite pseudointersection of $c_{s}$ 's. Then for every $s \in q^{\prime},\left[q_{s}^{\prime}\right]\left\lceil c\right.$ has a nonempty interior in ${ }^{c} 2$. By Lemma 3.3 there is $d \in[c]^{\omega}$ such that player I has a winning strategy in $\Gamma\left(\left[q^{\prime}\right], d\right)$. By Lemma 4.1, player I has a winning strategy in $\Gamma([q], d)$ and hence also in $\Gamma([p], d)$. This is a contradiction, since $[p] \in \mathfrak{C}_{2}$. Therefore $q=\emptyset$.

Hence $p=\bigcup\left\{\left(p_{n}\right)_{s_{n}}: n<\omega_{p}\right\}$. Let $\varphi_{n} \in \mathcal{F}$ for $n \in \omega$ be such that $\varphi_{n+1}(s)=$ $\varphi_{n}(s)$ for $|s| \leq\left|s_{n}\right|$ and (4.4) holds for all $n$. There is $\varphi \in \mathcal{F}$ that extends all $\varphi_{n}\left\lceil\left(p_{n}\right)_{s_{n}}\right.$, and then $[\varphi(p)]\left\lceil b=\bigcup\left\{\left[\varphi_{n}\left(\left(p_{n}\right)_{s_{n}}\right)\right]\left\lceil b: n \in \omega_{p}\right\}\right.\right.$ is countable (i.e., $s_{0}=\emptyset, \omega_{p}=1$, and $\left.p_{1}=\emptyset\right)$. This finishes the proof of Theorem 4.4

We do not know whether Theorem 4.4 can be generalized for Borel or even for analytic sets, and neither do we know whether condition (4.3) can be replaced by $(\exists \varphi \in \mathcal{F}) f_{\varphi}(A) \in \mathfrak{P}_{2}$. Let us define

$$
\mathfrak{P}_{2}^{*}=\left\{f_{\varphi}(A): A \in \mathfrak{P}_{2} \text { and } \varphi \in \mathcal{F}\right\} .
$$

Then $\mathfrak{P}_{2} \subsetneq \mathfrak{P}_{2}^{*} \subseteq \mathfrak{C}_{2}$. The next questions are open: Is $\mathfrak{P}_{2}^{*}=\mathfrak{C}_{2}$ ? Is $\mathfrak{P}_{2}^{*}$ an ideal? Is the $\sigma$-ideal generated by $\mathfrak{P}_{2}^{*}$ equal to $\mathfrak{C}_{2}$ ?

\section{REFERENCES}

[1] B. Balcar, J. Pelant, and P. Simon, The space of ultrafilters on $N$ covered by nowhere dense sets, Fund. Math. 110 (1980), no. 1, 11-24; MR 82c:54003

[2] M. Balcerzak and A. Rosłanowski, Coinitial families of perfect sets, J. Appl. Anal. 1 (1995), no. 2, 181-204; MR 97g:28001 
[3] J. Cichoń, A. Rosłanowski, J. Steprāns, and B. Wȩglorz, Combinatorial properties of the ideal $\mathfrak{P}_{2}$, J. Symbolic Logic 58 (1993), no. 1, 42-54; MR 94d:03098

[4] W. Dębski, J. Kleszcz, and Sz. Plewik, Perfect sets of independent functions, Acta Univ. Carolinae Math. Phys. 33 (1992), no. 2, 31-33; MR 95i:54014

[5] E. van Douwen, The integers and topology, Handbook of Set-Theoretic Topology (K. Kunen and J. E. Vaughan, eds.), North-Holland, Amsterdam-New York, 1984, pp. 111-167; MR 87f:54008

[6] J. Mycielski, Some new ideals of sets on the real line, Colloq. Math. 20 (1969), 71-76; MR 39:2934

[7] M. Repický, Permitted trigonometric thin sets and infinite combinatorics, Comment. Math. Univ. Carolinae 42 (2001), no. 4, 609-627; MR 2002k:03071

[8] A. Rosłanowski, On game ideals, Colloq. Math. 59 (1990), no. 2, 159-168; MR 92e:04004

Mathematical Institute of Slovak Academy of Sciences, Jesenná 5, 04154 Košice, SLOVAKIA

E-mail address: repicky@kosice.upjs.sk 